\title{
Learning a Model from Spatially Disjoint Data
}

\author{
Lawrence O. Hall, and Divya Bhadoria \\ Dept. of Computer Science and Engineering, ENB118 \\ University of South Florida \\ 4202 E. Fowler Ave. \\ Tampa, Fl 33620-9951 \\ hall@csee.usf.edu
}

\author{
Kevin W. Bowyer \\ Dept. of Computer Science and Engineering \\ University of Notre Dame \\ South Bend, IN 46556 \\ kwb@cse.nd.edu
}

\begin{abstract}
Some large-scale simulations are distributed over thousands of processors and generate terabytes of data. The output may take weeks or months to debug and explore. Therefore, learning a model that allows users to quickly focus on interesting events would be a great timesaver. Training data will not fit in one physical memory and the most natural splitting of the data into tractable size subsets would be along lines of disk farms, causing data for simulated objects to be split across training sets. In general, the training sets will contain very few interesting examples. A $k$ nearest centroids approach was developed to classify unseen data. ROC analysis on a set of face images (partitioned spatially) indicates that this is a promising approach for the larger problem.
\end{abstract}

Keywords: nearest neighbor, learning, spatially disjoint data, skewed data.

\section{Introduction}

There are important application contexts in which it is not reasonable to construct a classifier from all of the available training data. One such application context occurs in the DOE's ASC program [1]. Massive simulation data sets are created on a parallel computer, recording the simulation of important physical processes underlying some hypothetical real-world event. Currently, experts visually browse the dataset for interesting events in the simulation. In this context, an "interesting event" may be the beginning or end of some phenomenon in the simulation, or it may in effect indicate a bug in the simulation model. This sort of manual browsing for interesting events in the simulation is of course time-consuming. Therefore, one would like to construct a classifier that could automatically label the interesting events.

This problem has important and interesting aspects that make it distinct. One aspect is that partitions that are spatially homogeneous with respect to the physical model generally result in class distributions that are widely varying across partitions. For example, a simulation might record the deformation and rupture of a fifty-five gallon metal drum over the time sequence of some event. In this case, each processor may hold the representation of part of the drum that was in a given volume of 3D space at the outset of the simulation. Thus the data at a given processor will generally not be representative of the data for the whole simulation. In terms of the pattern recognition context, the different partitions of the training data may have highly unequal distributions of the various classes. Another problem is that interesting events are, pretty much by definition, rare [4]. For many partitions of the data and most time steps of the simulation, there might be no interesting event that occurs.

The problem is to find a good way of constructing a classifier, or committee of classifiers, from: (1) a large dataset, (2) that is partitioned in some way that is natural to the underlying application, but may result in a very unequal class distribution across partitions, and (3) where most of the data represents the least interesting class. Properties (2) and (3) are what distinguish this problem from typical pattern recognition applications. Typically, when a set of training data is partitioned, it is assumed that the relative class distribution is at least similar across partitions. We know of no other work that focuses specifically on the problem of how to cope with partitions that may present pathological variations in class distribution. The problem is made more acute in this case because the class of interest is a minority class.

In this paper, we explore this problem context using face images from the FERET database [7], [10]. The images are spatially broken into regions and classifiers are constructed to recognize interesting, somewhat interesting, and uninteresting areas. We report on a nearest centroid approach and compare it to learning a decision tree on all the data. These preliminary results indicate that it may be possible to provide good guidance for large-scale simulation developers and users. An ROC curve analysis of the tradeoff of true positives for false positives is also given.

\section{${ }^{*}$ 0-7803-8566-7/04/\$20.00 @ 2004 IEEE.}




\section{Data}

The FERET image corpus has been widely used in testing and evaluation of face recognition algorithms using standardized procedures [7], [10]. The final corpus consists of 14,051 eight-bit grayscale images of human faces representing different lighting conditions and facial expressions. The $\mathrm{F}_{\mathrm{A}}$ images are frontal views with standard lighting and neutral facial expression. The $\mathrm{F}_{\mathrm{B}}$ images are frontal views with standard lighting and an alternate facial expression, typically a smile.

\subsection{Creating Test and Train Sets}

To create the train and test sets, face images from five persons were chosen at random from the FERET database. For each person, two images were selected, one $\mathrm{F}_{\mathrm{A}}$ and one $F_{B}$, for a total of ten images. One image of each face constituted the training set and the other formed the test set. In this way, we formed train and test sets of five images each such that all faces are presented in both sets. The images are not shown here due to space limits, but can be found in Bhadoria's thesis [2].

\subsubsection{Pre-processing}

Before features were extracted from the images, histogram equalization was performed to normalize illumination across the images. Also, the images were scaled and translated so that eye centers were aligned to fixed pixel locations in a 130x150 standard image size. An elliptical mask was applied to gray out the background, leaving only the face. These steps help to ensure that our model is applicable to different images.

We defined class boundaries by coloring regions to represent levels of interest. Boundaries were drawn free hand, using a freely distributed image manipulation package called GIMP [11]. We created a three-class problem. The eyes and mouth were in the interesting class, the eyebrows were labeled somewhat interesting, and everything else was labeled uninteresting. Clearly, the interesting class has a mixture of objects, perhaps making it more realistic. Figure 1 shows an example of a training and test image after preprocessing and labeling.

For this work, we created the following combination of six local and point features for our feature vectors [3], [9], [12]. None of them require object detection. They were: the intensity value of the pixel of interest, maximum intensity over a fixed neighborhood around the pixel of interest, minimum intensity over a fixed neighborhood around the pixel of interest, intensity range computed as the difference between maximum and minimum intensities over the fixed neighborhood around the pixel of interest, arithmetic mean of intensity values over the neighborhood, and standard deviation of intensities over the neighborhood. A $5 \times 5$ window was used for the region-based features.
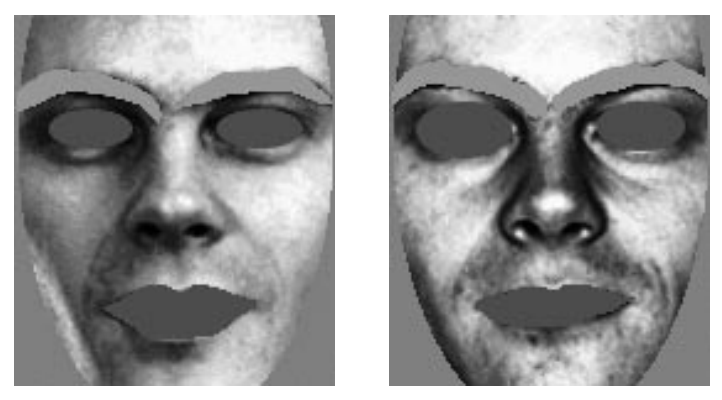

Figure 1. On the left is a training image after preprocessing and region labeling, with red indicating very interesting, green indicating somewhat interesting, and everything else marked uninteresting; On the right is a test image of the same person. Note in $\mathrm{B} / \mathrm{W}$ the face is one medium gray class and the eyes and mouth are a darker gray with the eyebrows a lighter gray.

Approximately $84 \%$ of the pixels in the images belong to the not interesting (NI) class, $11 \%$ to the interesting (I) class, and $5 \%$ to the somewhat interesting class (SI). Class imbalance this extreme or greater is an inherent element of the application of interest. Almost by definition, only a small fraction of the data generated in a real world simulation experiment would fall in the I or SI class, so we believe this is a realistic aspect of our experimental data. It also means that one can obtain a reasonably good sounding $84 \%$ accuracy by simply guessing everything is not interesting. We intend to show more useful results.

The training data was next divided into partitions that are spatially homogeneous with respect to the data. Each face image might be divided into eight equal-size rectangular regions, by cutting the image into two columns and four rows. Thus we get $5 \times 8=40$ spatial regions. Note that some partitions may have a pathological distribution of training data. In our example training data, 4 of these spatial regions have purely NI data, 27 have some MI data, 12 have some SI data, and only 3 partitions have all three classes of data.

\section{Classifiers}

We used a variant of $k$-nearest neighbor classifier $(\mathrm{KNN})$ that we call a $k$-nearest centroid classifier (KNC). First, centroids are calculated for each class present in every region. Together, these centroids form the "train set". The classifier then finds the $k$-nearest centroids for the pixel to be classified and these centroids are then allowed to vote for 
the pixel class. In the simplest case, if $k=1$, then the test pixel will be assigned the class represented by the centroid to which it is closest. KNN has some advantages in that the training is simple and it provides reasonable accuracy [6]. Using centroids helps reduce the testing time significantly. To obtain a baseline result for comparison purposes, the C4.5 decision tree learning software was utilized [8].

\section{Experiments}

We have 40 spatial regions from the 5 training images. For each of these spatial regions, a centroid was calculated for every class present in it. Since the NI class is present in all 40 spatial regions, we get 40 centroids belonging to the NI class. Class I is present in 27 spatial regions, resulting in 27 centroids for the I class and finally, the SI class is present in only 12 spatial regions so we get 12 SI centroids. Thus we get a total of 79 centroids. Now for each test image, pixel classifications are made using $\mathrm{KNC}$ for $k=1$, 3 ....n where $n$ is the number of centroids representing the smallest minority class. Allowing any more centroids to vote is very likely to affect the accuracy for the minority class since the number of votes in their favor will always be insufficient. The smallest minority class here is SI, with 12 centroids. As a general rule, even numbers are avoided in nearest neighbor classification. Thus, we do KNC for $k=$ $1,3,5,7,9$ and 11 . Ties were broken in favor of the more interesting class, with the order of preference I > S I > NI.

\subsection{Results}

The classification accuracies of the KNC classifier for two of the five test images are presented in Figure 2. They are an average and worst case. The results are presented for the individual classes since a high overall accuracy is a very misleading term in this context. Our aim here is to learn the regions of interest in data, even if in doing so we may have to lose some accuracy on the not interesting data. The idea is that if we do not completely miss any regions of interest (I and SI) then having false negatives is not an insurmountable problem. Further, any false positives in the vicinity of the interesting regions will be acceptable since they will tend to lead to the right place. Besides, confusing I and SI with each other is far more acceptable than confusing either of them with NI.

The charts in Figure 2 give the accuracy for the three classes as well as the combined accuracy of the I and SI classes. This combined accuracy tells us how well our classifier was able to differentiate any interesting event from an event of no interest to us.

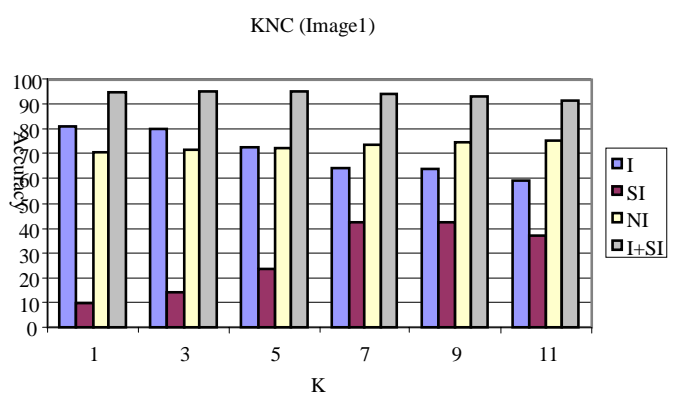

a

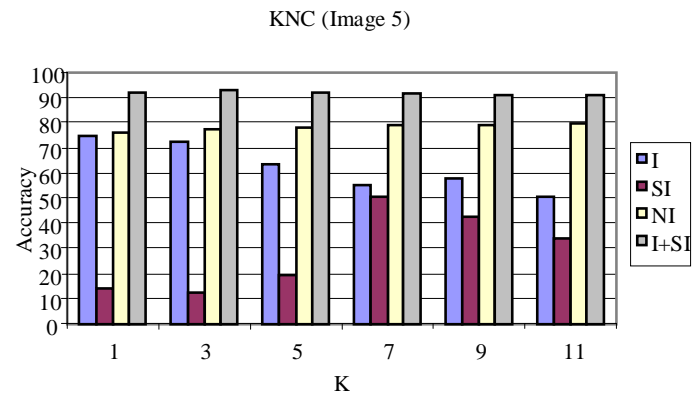

$b$

Figure 2. a) One of the most accurate images in terms of I+SI and it is the person from Fig. 1. b) Better on not interesting NI.

The accuracy histograms of Figure 2 show that the $\mathrm{KNC}$ classifier is very well able to achieve this goal. The combined percent accuracy of I and SI is almost always in the high 90s. The accuracy for the NI class is also high. This means that the classifier did not greatly confuse the NI class with either of the I or SI classes. This also tells us that while the classifier did not greatly confuse NI with any other class, it did, however, get heavily confused between the I and SI examples. The low SI class accuracy and high I can be partly attributed to the tie-breaking rule we have used. Many times when the classifier got a non NI test example for classification, there was a tie and since the tie breaker rule has a preference for the I class, these pixels get classified as I. This resulted in a high number of false positives, bringing down the SI class accuracy. To check this analysis, a quick experiment was run by changing the tie breaker rule to give higher preference to the SI class. That is, if we have the order of preference as SI $>$ I $>$ NI, we get similar results except this time the SI accuracy was higher as we increased K. For two of the five test images the SI class accuracy was higher than for class I. There was still significant confusion between the two classes.

In Figure 3a, we show the baseline results from a default pruned decision tree trained on all of the training data. Figure $3 \mathrm{~b}$ shows the $\mathrm{KNC}$ results for the same image (the test image from Fig. 1). There are more tightly clustered false positives in the KNC result Figure (3b). The 
distributed approach certainly results in not much worse classification. Generally, the nose, especially nostrils, tends to get misclassified into an interesting class. This particular image does not have hair on the forehead, but those that do have that misclassified and this seems understandable given that eyebrows will be similar with our rather primitive set of features.

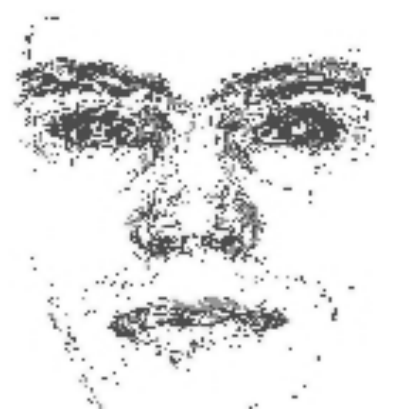

$\mathbf{a}$

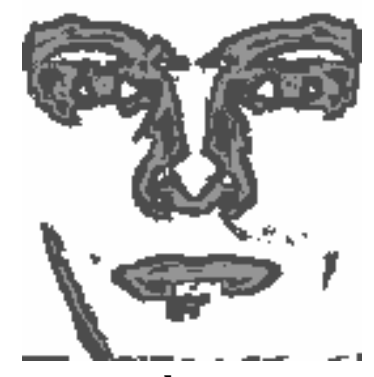

b
Figure 3. a) Baseline C4.5 result b) Result of KNC. In $\mathrm{B} / \mathrm{W}$ lighter gray is I and darker is SI, NI is white.

\subsection{ROC Curves}

Receiver-Operator Characteristic (ROC) curves [5] plot false-positive (FP) rates on the $\mathrm{x}$-axis and true-positive (TP) rates on the $y$-axis. The ROC curve identifies how many false positives one must tolerate to be guaranteed a certain percentage of true positives. An ideal ROC curve is the step-function.

In Figure 4, classes I and SI have been combined together as the interesting class. A total of 79 centroids were used (one centroid per class per region). The points on the curve were obtained as follows: K-nearest $(\mathrm{k}=1,2, \ldots, 79)$ centroids were used to vote for the test class. Ties were broken in favor of the majority class. The TP and FP rates were highest for $k=1$. As $\mathrm{k}$ was increased, the points moved towards zero. This was because the number of interesting centroids was too small (39 out of 79) so as the number of voting centroids was increased, more and more votes start coming for the not interesting class. After a certain value of $k$, the vote will always be in favor of the not interesting class. For every test pixel, its distance from all centroids was calculated. Then $\mathrm{k}$ nearest centroids were looked at, for $1 \leq k \leq 79$. If even one of them voted in favor of the interesting class, the test pixel was classified as interesting. So as the value of $k$ increased, more and more TP and FP were created as the likelihood of encountering an interesting centroid increased. This method is exactly similar to the one above for $k=1$, which forms the bridge point between the two methods

The single decision tree trained on all the data is $49.5 \%$ accurate on all the test images with a $5 \%$ false positive rate. So, it is slightly more accurate on average at a lower false positive rate than any of the KNC classifiers for individual images. However, it did see all the training data.

\section{Summary and Discussion}

We have simulated having large amounts of simulation data by using face images from the FERET database and breaking them into spatial regions. The presented experimental results show that it is possible to achieve high recognition rates of interesting regions even when the data for training was broken up in non-intuitive ways. That is, adjacent regions may be given to different classifiers. In this work, we have only shown results from a nearest neighbor type of classifier. The use of centroids of classes in regions simplifies the testing procedure, by minimizing the number of vectors for testing. It is able to deal with the fact that not all regions will have all of the classes in them. Some regions may have only one class. In general, the data labeled interesting will be significantly less than the not interesting data.

It has been shown how to get a trade-off (for the two class problem) in terms of true positives and false negatives. One issue that is not addressed is where the false positives lie. If they are scattered all over the data (image in this case), this would cause someone to look at many regions erroneously. Most of the false positives are around the nostrils and hair at the top of the head, which, for face images anyway, are quite like the training data. The nostrils, which are dark regions, match the eyes to a degree. Clearly hair and eyebrows are very similar.

We are utilizing more sophisticated classifiers on the training data and looking at how to minimize the false positives. Truly it is not necessary to recognize that much of an interesting region, simply to point the developer to it. In summary, these preliminary results are encouraging. 


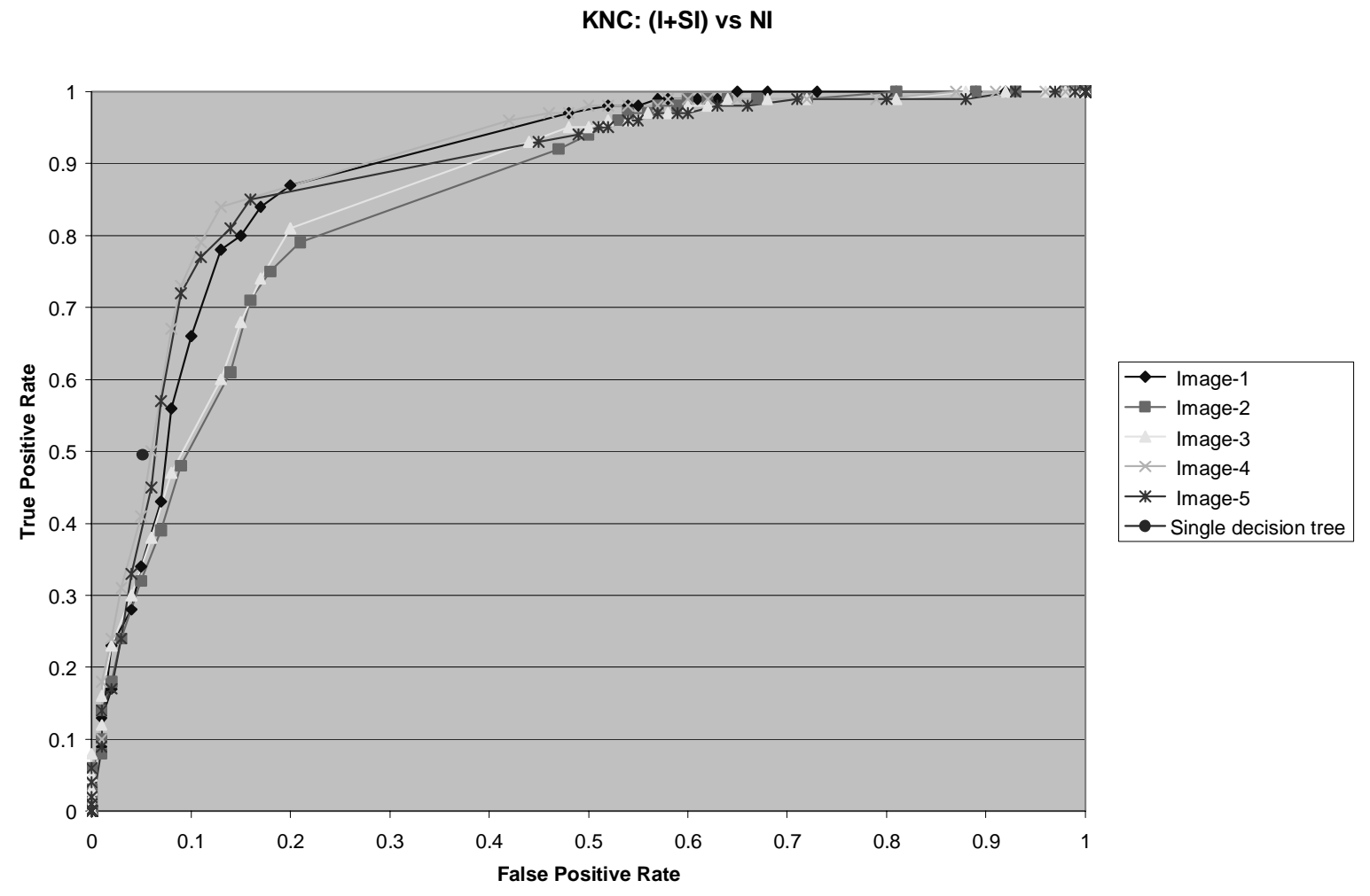

Figure 4. ROC curves: K-nearest centroids and Decision tree.

Acknowledgements: This research was partially supported by the Department of Energy through the ASCI Views Data Discovery Program, Contract number: DEAC04-76DO00789. Thanks to Robert Banfield for the classified decision tree face image and Larry Shoemaker for the classified face image from KNC. Portions of the research in this paper use the FERET database of facial images collected under the FERET program

\section{References}

[1] Advanced Simulation and Computing program Department of Energy,

(http://www.nnsa.doe.gov/asc/home.htm).

[2] D. Bhadoria, Learning from Spatially Disjoint Data, M.S. Thesis, Univ. of South Florida, Tampa, 2004.

[3] S. Brand, J. Laaksonen, E. Oja, "Statistical Shape Features in Content-Based Image Retrieval", International Conf. on Pattern Recognition, 2000.
[4] N. Chawla, K. Bowyer, L. Hall, P. Kegelmeyer, SMOTE: Synthetic Minority Over-Sampling Technique, Journal of Artificial Intelligence Research, vol. 16, 321357, 2002.

[5] J.A. Hanley, B.J. McNeil, "The meaning and use of the area under the Receiver Operating Characteristic (ROC) curve", Radiology, 143, pp. 29-36, 1982.

[6] T.M. Mitchell, Machine Learning. New York, NY: McGraw Hill, 1997.

[7] P. J. Phillips, H. Wechsler, J. Huang, and P. Rauss, "The FERET database and evaluation procedure for face recognition algorithms," Image and Vision Computing Journal, Vol. 16, No. 5, pp 295-306, 1998.

[8] J.R. Quinlan, C4.5: Programs for Machine Learning. San Francisco, CA: Morgan Kaufmann, 1993

[9] M. Sonka, V. Hlavac, R. Boyle, Image Processing, Analysis and Machine Vision: Kluwer Academic Publishers, 1998. 
[10] "The Facial Recognition Technology (FERET)

Database", http://www.itl.nist.gov/iad/humanid/feret/

[11] "The GIMP”, http://www.gimp.org/

[12] M. Tuceryan and A.K. Jain, Texture Analysis. The Handbook of Pattern Recognition and Computer Vision (2nd Edition), pp. 207-248: World Scientific Publishing Co., 1998. 